Forum $2021 \cdot 36: 71-73$

https://doi.org/10.1007/s12312-020-00873-1

Online publiziert: 3. Dezember 2020

(c) Springer Medizin Verlag $\mathrm{GmbH}$, ein Teil von Springer Nature 2020

\author{
S. Hüning' $\cdot$ U. Beiteke' $\cdot$ P. Dücker' $\cdot$ L. Tizek² $\cdot$ A. Zink ${ }^{2} \cdot$ D. Nashan' \\ 'Hautklinik, Klinikum Dortmund gGmbH, Dortmund, Deutschland \\ ${ }^{2}$ Klinik und Poliklinik für Dermatologie und Allergologie am Biederstein, Technische Universität München, \\ München, Deutschland
}

\title{
Corona-Pandemie, ein Risikofaktor für die zeitnahe Versorgung von Hautkrebspatient?
}

\section{Versorgung onkologischer Patienten während der Corona-Krise in einem Hauttumorzentrum}

\begin{abstract}
In den letzten Monaten stand vielfach die Frage im Fokus, ob es während der Corona-Pandemie bedingt durch die Verschiebung von Ressourcen zu Versorgungsengpässen und insbesondere auch zur eingeschränkten Versorgung onkologischer Patient kommt. Dazu gehören Vor- und Nachsorgeuntersuchungen, diagnostische Sicherung von Tumoren, zügige Operationen und notwendige Systemtherapien, um einem Tumorprogress und dem Fortschreiten von Tumorerkrankungen und damit einhergehender erhöhter Mortalität vorzubeugen.
\end{abstract}

\section{Studienaufbau und -ergebnisse}

Im Rahmen der Qualitätssicherung im zertifizierten Hauttumorzentrum (HTZ) am Klinikum Dortmund wurde eine Befragung onkologischer Patient der Hautklinik Dortmund durchgeführt, um die Vorgehensweise und Behandlung im Rahmen der Corona-Pandemie aus persönlicher Sicht der Betroffenen $\mathrm{zu}$ erfassen.

Vom 16. Juni bis 16. August 2020 wurden dazu insgesamt 163 Fragebögen an onkologische Patient ausgeteilt, davon 50 stationär und $113 \mathrm{im}$ ambulanten Bereich, welche die Patient selbstständig ausfüllten. Durch gravierendes fehlerhaftes Ausfüllen seitens der Patienten waren für die statistische Auswertung lediglich 153 Fragebögen verwertbar.

Von den eingeschlossenen 153 Patient wurden 54,04\% aufgrund eines Melanoms im HTZ behandelt, gefolgt von Patient mit „nonmelanoma skin cancer“ (NMSC) in $24,84 \%$ und mit primär kutanen Lymphomen in 10,56\% der Fälle. Keine Angaben zur Tumorentität machten $10,56 \%$ der Patient (• Abb. 1).

Im Gesamtkollektiv wurde die Verschiebung oder Absage von geplanten Terminen im HTZ von $6,37 \%$ als selbstständige Absage durch Patient und von $12,10 \%$ als Terminverschiebung durch das HTZ notiert. Das Corona-adaptierte Hygienekonzept des Klinikums Dortmund beinhaltet neben standardisierten Maßnahmen wie Abstandsregeln, Mundschutz und Desinfektion, angemeldete und kontrollierte Patientenzugänge, gesteuerter Patientenfluss, Verteilung der Patient bei i.v.-Therapien platzadaptiert auf mehr Räume wie auch negative COVID-19-Abstriche vor stationärer Aufnahmen oder im Akutfall Vergabe von Einzelzimmern. Das damit einhergehende subjektive Sicherheitsempfinden wurde von den Patient als sehr positiv reflektiert: $89,80 \%$ fühlten sich während der
Corona-Pandemie „sehr sicher im Klinikum Dortmund“.

Nur ein einziger Patient von den insgesamt 63 Patient, die mit einer onkologischen Systemtherapie behandelt wurden, berichtete, dass es aufgrund eines innerfamiliären Corona-Falls zu einer kurzzeitigen Verschiebung des Therapiezyklus kam. Es gab keine Corona-bedingten Therapieabbrüche.

Betrachtet man diese Ergebnisse, war zunächst davon auszugehen, dass es im HTZDortmund durch die Corona-Situation zu keiner signifikanten Verschlechterung in der klinischen Anbindung und Versorgung sowohl ambulanter als auch stationärer onkologischer Patient kam.

Die Frage war zu ergänzen, ob der allgemeine primäre Zugang in die Klinik im gleichen Maße genutzt wurde. Aufgrund des tumorstadienadaptierten Mortalitätsrisikos wurde eine Analyse zur Subgruppe der Melanompatient angeschlossen.

Vergleicht man die Anzahl der Melanomerstdiagnosen im 1. Halbjahr 2019 mit der im gleichen Zeitraum 2020, zeigt sich eine Reduktion der Primärdiagnosen von 86 auf 62. Insbesondere die Anzahl neu diagnostizierter Melanome im Stadium IA und IB (American Joint Committee on Cancer 2018) zeigte in unserem HTZ einen deutlichen Rückgang. 


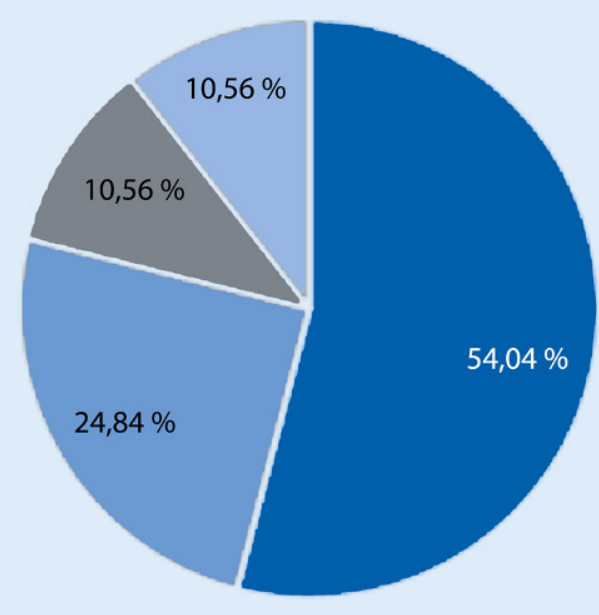

Melanom NMSC Lymphom n.a.
Abb. $1 \triangleleft$ Tumorentitäten der befragten onkologischen Patient $(n=153)$. NMSC "nonmelanoma skin cancer"

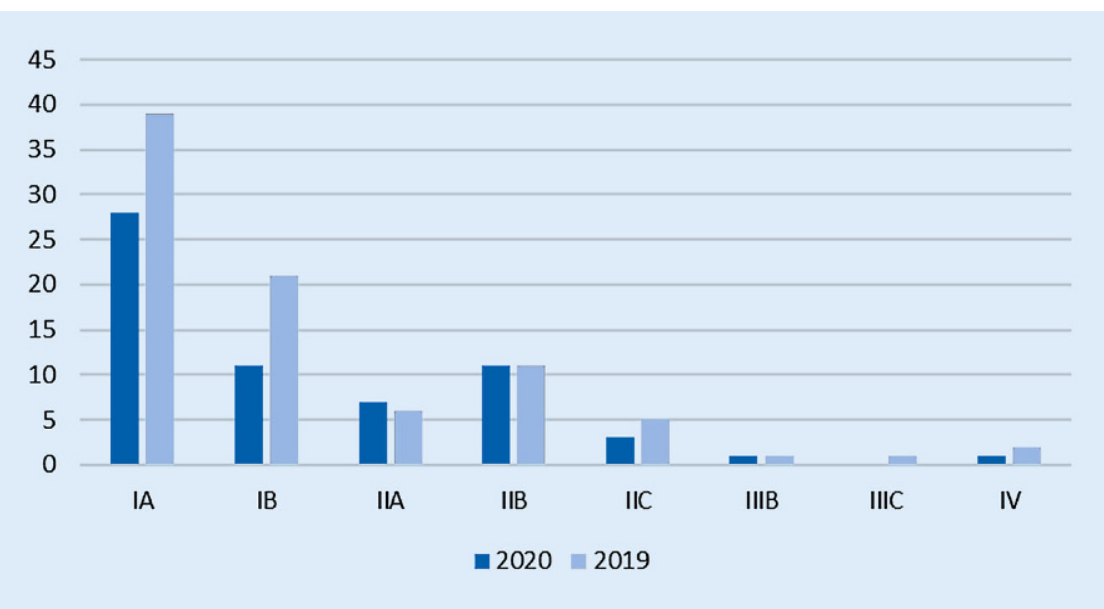

Abb. $2 \Delta$ Anzahl der Erstdiagnosen von malignen Melanomen im HTZDortmund im 1. Halbjahr 2019 und $2020 \mathrm{im}$ Vergleich

Im Jahresvergleich war eine Reduktion um $28,21 \%$ von Patient im Stadium IA (Tumordicke $[\mathrm{TD}] \leq 1,0 \mathrm{~mm}$ ) und um $47,62 \%$ von Patient im Stadium IB (TD $\leq 2,0 \mathrm{~mm}$ ) zu verzeichnen (• Abb. 2). In den fortgeschrittenen Tumorstadien blieben die Primärfallzahlen weitgehend stabil. Geht man von einer gleichbleibenden oder sogar steigenden Inzidenz an Melanomerkrankungen aus [1], resultiert aus o. g. Daten, dass ein Teil der Melanompatient in diesem Jahr bislang nicht diagnostiziert bzw. in der Klinik behandelt wurde.

Zur Frage der Diagnosestellung und mit Blick auf niedergelassene Kolleg

lich zur Optimierung von Marketingkampagnen entworfene Google Ads Keyword Planner kann, wie andere Publikationen zeigen, gut für wissenschaftliche Zwecke und derartige Fragestellungen genutzt werden [2,3]. Das Suchvolumen der Bevölkerung zum Themenkomplex „malignes Melanom“ im Raum Dortmund war damit zu überprüfen. $\mathrm{Zu}$ nächst wurden die am häufigsten mit dem Begriff „malignes Melanom" assoziierten Stichwörter $(n=152)$ identifiziert und anschließend monatliche Auswertungen dieser Suchwörter erstellt. Vergleicht man nun erneut das 1 . Halbjahr 2019 mit dem 1. Halbjahr 2020, fällt auf, dass im Halbjahr 2020 insgesamt 1280 Suchanfragen mehr zum Melanom erfolgten als 2019 (2019: 4100; 2020: 5380) (• Abb. 3).

\section{Zusammenfassung und Diskussion}

Zusammenfassend resultiert aus unseren Recherchen, dass bei gleichbleibend guter Versorgung der bereits angebundenen onkologischen Patient im HTZ sich eine geringere Patientenanzahl mit der Erstdiagnose Melanom vorstellte. Eine ebenfalls geringere Anzahl an durchgeführten Hautkrebsscreenings im niedergelassenen Bereich und steigende Google-Recherchen zum Thema Melanom verdeutlichen eine Verschiebung in private Bereiche.

Damit ist eine Corona-assoziierte $\mathrm{Zu}$ rückhaltung in puncto Screening und Tumordiagnose belegt. Dies spiegelt sich auch im COVID-19 Snapshot Monitoring (COSMO) der Universität Erfurt wider. In regelmäßigen Befragungen der Bevölkerung zur Wahrnehmung der Corona-Pandemie wurde im Rahmen der 17. Welle (KW 10-30, Stand 24.07.2020) beschrieben, dass jeder 9 . Befragte (11\%) seit März 2020 eine Krebsvorsorgeuntersuchung wegen Corona aufgeschoben hat. Dieser Trend ist insbesondere bei älteren Patient erkennbar [4]. Dies ist umso nennenswerter, weil die Gruppe der „Älteren“ gleichzeitig die höchste Zahl der von Hauttumoren Betroffenen darstellt.

In einer Onlineumfrage des Berufsverbandes der Deutschen Dermatologen (BVDD) gaben 38,3\% der Dermatolog 


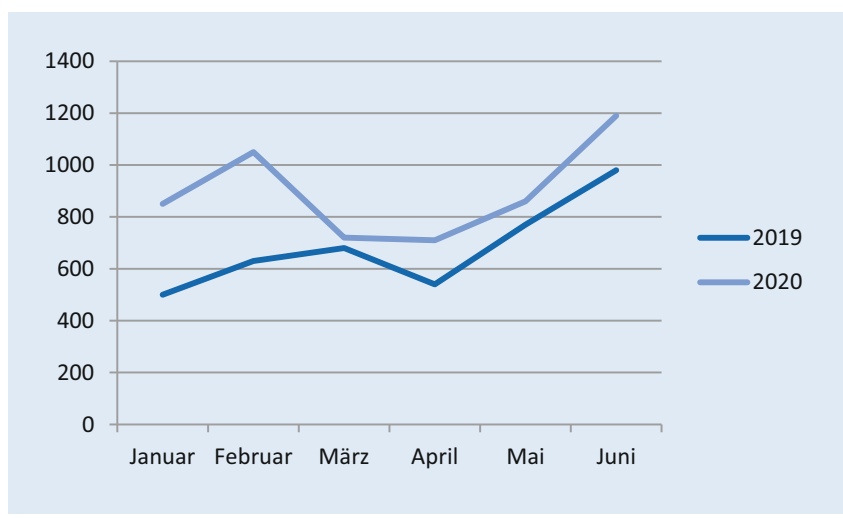

Abb. $3<$ Trends im Google-Suchvolumen zum malignen Melanom in Dortmund im 1. Halbjahr 2019 und $2020 \mathrm{im}$ Vergleich $(n=530)$ an, dass Prävention im Sinne des Hautkrebsscreenings seitens der Patient weniger nachgefragt worden sei, und 13,5\% der Dermatolog haben ihrerseits Präventionsleistungen nur eingeschränkt angeboten [5]. Das Zentralinstitut für kassenärztliche Versorgung in Deutschland beziffert zeitweilig bis zu $71 \%$ reduzierte Hautkrebsscreenings gegenüber dem Vorjahr [6]. Ob und inwieweit verspätete Diagnosen und damit schlechtere Prognosen aus dieser Zeit resultieren, bleibt zu untersuchen.

Versorgungskapazitäten für CoronaErkrankte vorzuhalten, mag $\mathrm{zu}$ einer allgemeinen Zurückhaltung beigetragen haben. Aber auch direkte Aufforderungen an die Ärzt, wie beispielsweise in einem Telegramm der KVWL vom 17.03.2020, mögen den Zugang gehemmt haben. Es wurde dazu aufgerufen, dass Termine die „nicht mit höchster Priorität abgearbeitet werden müssen, zum Beispiel Vorsorgeuntersuchungen " abgesagt werden, um ausschließlich dringende $\mathrm{Pa}$ tientenkontakte zu ermöglichen. Durch diese Diktion sind Absagen und $\mathrm{Zu}$ rückhaltung der niedergelassenen Ärzt begründet und verständlich. Erst am 30.04.2020, also 6 Wochen später, wurde empfohlen, die Praxen langsam wieder für Elektivuntersuchungen zu öffnen [7].

Diese Szenarien unterstreichen die Fallzahlentwicklung des HTZ Dortmund und lassen vermuten, dass insbesondere asymptomatische Tumorpatient bzw. Patient in Frühstadien zeitverzögert vorstellig werden.

Zugangsbeschränkungen, Angst, neue Wertung von Krankheitsdringlichkeiten, ständig wechselnde Reglements, unzureichende Kommunikation und fehlen-
3. Tizek L, Schielein MC, Rüth M et al (2019) Interest in skin cancer in urban populations: a retrospective analysis of google search terms in nine large german cities. Acta Derm Venereol. https://doi. org/10.2340/00015555-3214

4. Betsch C (2020) Ergebnisse aus dem COVID-19 SnapshopMOnitoring COSMO:Diepsychologische Lage (17. Welle, KW 10-30)

5. Ärzte Zeitung. Wie Corona die Versorgung in den Hautarztpraxen verändert. 10. Aug. 2020. 2020:5.

6. Ärzte Zeitung (Hrsg) (2020) So hat sich die Corona-Pandemie auf die Arztpraxen ausgewirkt. 27.07.2020. https://www.aerztezeitung. de/Politik/Aerzte-haben-sich-bei-Corona-nichtweggeduckt-411619.html?bPrint=true. Zugegriffen. 26. Okt. 2020

7. KVWL (2020) Telegramm Corona-Krise: Praxisbetrieb anpassen Bd. 48, S1-2 des Verständnis haben sicher zu dieser Entwicklung beigetragen. Die bisher erhobenen Zahlen sollten uns daher sensibilisieren, dass Vorsorgeuntersuchungen weiterhin im Fokus bleiben müssen und der notfallmäßige Zugang von Patient mit Verdacht auf einen Hauttumor gefördert werden und immer möglich sein muss. Ein Appell an die Bevölkerung, um dem so berechenbaren Anstieg dickerer Melanome entgegenzutreten, wäre wünschenswert.

\section{Korrespondenzadresse}

\section{Dr. med. S. Hüning}

Hautklinik, Klinikum Dortmund gGmbH

Beurhausstr. 40, 44137 Dortmund, Deutschland svea.huening@klinikumdo.de

\section{Einhaltung ethischer Richtlinien}

Interessenkonflikt. S. Hüning, U. Beiteke, P. Dücker, L. Tizek, A. Zink und D. Nashan geben an, dass kein Interessenkonflikt besteht.

Für diesen Beitrag wurden von den Autoren keine Studien an Menschen oder Tieren durchgeführt. Für die aufgeführten Studien gelten die jeweils dort angegebenen ethischen Richtlinien. Von allen an der Befragung Beteiligten liegt eine schriftliche Einverständniserklärung vor. Die Zustimmung einer Ethikkommission war nicht notwendig.

\section{Literatur}

1. Leitlinienprogramm Onkologie, Deutsche Krebshilfe, Deutsche Krebsgesellschaft, AWMF. S3Leitlinie Diagnostik, Therapie und Nachsorge des Melanoms. Leitlinienprogr Onkol. 2019:1-81.

2. Zink A, Schuster B, Rüth M et al (2019) Medical needs and major complaints related to pruritus in Germany: a 4-year retrospective analysis using Google AdWords Keyword Planner. J Eur Acad Dermatol Venereol. https://doi.org/10.1111/jdv. 15200 